\title{
STUDY OF DIFFUSION IN LATTICE-GAS FLUIDS AND COLLOIDS
}

\author{
Martin A. van der Hoef and Daan Frenkel \\ F.O.M.-Institute for Atomic and Molecular Physics \\ Kruislaan 407, 1098 SJ Amsterdam, The Netherlands
}

\begin{abstract}
We describe in detail two techniques that have been developed to study diffusion phenomena in lattice-gas fluids, and in hybrid lattice gas-colloidal systems.
\end{abstract}

\section{Introduction}

Both lattice gas models and cellular automata have been around for many years. However, it was only in 1986 that Frisch, Hasslacher and Pomeau introduced a Lattice Gas Cellular Automaton(LGCA) that could be used to model a hydrodynamic fluid [1]. The hope was that these models could be more efficient than traditional methods in the study of complex flow phenomena, e.g. flow at high Reynolds number. The advantage of LGCA compared with other methods is that they are numerically stable, and flow in systems with complex boundary conditions is not more difficult to study than flow in more simple geometries. A drawback of LGCA is that they are inherently noisy, and averaging over this statistical noise requires massive calculations. However, LGCA can also be used for a quite different purpose, namely as a model system in which fundamental concepts in kinetic theory can be tested. Lattice gas models provide a simplified description of the microscopic dynamics of atomic fluids, yet most of the 'interesting' correlations are intrinsically present. The simplicity of LGCA facilitates not only the simulations but also the theoretical analysis of transport phenomena in such systems. This makes LGCA an ideal tool to test the basic assumptions that are made in various approximate theories of transport in fluids. Another application of LGCA, initiated by Ladd, Colvin and Frenkel [2], is to use it as a model for the solvent in colloidal suspensions. In this context LGCA are considered as the computationally cheapest model that has the essential properties of the solvent, namely hydrodynamic interactions and random (brownian) forces. The idea behind this approach is that it might be feasible to do simulations of colloidal systems in which both the microscopic scales and the mesoscopic scales are simultaneously present. Techniques that have 
been developed for both type of studies will be discussed in this lecture. First we show how one can measure the velocity autocorrelation function in a lattice gas with very high accuracy, in order to make detailed comparisons with the kinetic theories. Subsequently, in section four, we describe a technique to model the motion of a colloidal particle in the LGCA-fluid.

\section{Definitions}

Let us first briefly summarize the essentials of lattice gas cellular automata, in order to establish the notation that is used in subsequent sections. In what follows, we assume that the reader is familiar with the basics of lattice-gas models. For more details on this subject the reader is referred to the original papers on lattice gases $[1,3,4]$, and also to the contributions of M.H. Ernst and J.-P. Boon in these proceedings.

In LGCA-models, time and space are discretized. This means that the model system is defined on a lattice and the state of the automaton is only defined at regular points in time with separation $\Delta t$. At these discrete times, particles are situated at the lattice nodes, with $b$ possible velocities $\boldsymbol{c}_{\boldsymbol{i}}, i \in\{1,2, \ldots, b\}$. The set $\mathbf{c}_{\boldsymbol{i}}$ can be choosen in many different ways, although they are restricted by the constraint that

$$
\mathrm{r}^{\prime}=\mathrm{r}+\mathrm{c}_{i} \Delta t
$$

where $\mathbf{r}$ and $\mathbf{r}^{\prime}$ must be lattice nodes. This ensures that the particle stays on the lattice. We can imagine that a particle at node $\mathbf{r}$ with velocity $\mathbf{c}_{i}$ occupies a link connecting the node $\mathbf{r}$ with the node $\mathbf{r}^{\prime}$. In most cases, the velocities are such that only nearestneighbor lattice nodes can be reached in a timestep. No two particles can be at the same lattice node with the same velocity. The time evolution of the LGCA consists of two steps:

1. Propagation: All particles move in one timestep $\Delta t$ from their initial lattice position $r$ to a new position $\mathbf{r}^{\prime}=\mathbf{r}+\mathbf{c}_{i} \Delta t$.

2. Collision: The particles at all lattice nodes undergo a collision that conserves the total number of particles and the total momentum at each node. The collision rules may or may not be deterministic.

For convenience we work in reduced units with the mass of the lattice-gas particles, the distance between near-neighbor sites, and the time step $\Delta t$ for updating the lattice gas all equal to unity. The state of the the automaton at time(-point) $t$ is completely given by $s_{i}(\mathbf{r}, t)$, which is equal to $1(0)$ if a particle is present(absent) on node $\mathbf{r}$ with velocity $\mathbf{c}_{\boldsymbol{i}}$. The local microscopic mass density is defined as

$$
\rho(\mathrm{r}, t)=\sum_{i} s_{i}(\mathrm{r}, t)
$$

which is simply the number of particles at node $\mathbf{r}$. The local microscopic velocity field $u_{x}(\mathbf{r}, t)$ is defined by

$$
\rho(\mathbf{r}, t) u_{x}(\mathbf{r}, t)=\sum_{i} s_{i}(\mathbf{r}, t) c_{i x}
$$

Both density and velocity are conserved locally in collisions. In order to obtain macroscopic relations, one can go through the the conventional procedure of statistical mechanics, and perform ensemble averaging. This approach is basically the same for 
lattice gas fluids and ordinary fluids, and can be found in [3]. A first step is to define the one-particle distribution function

$$
f_{i}(\mathrm{r}, t)=\left\langle s_{i}(\mathbf{r}, t)\right\rangle
$$

where $\langle.$.$\rangle denotes ensemble averaging. If one assumes local equilibrium, then the$ corresponding one-particle distribution function proves to be of the Fermi-Dirac type. This function can be expanded in powers of the local velocity $\mathbf{u}$, provided that $u$ is small. The result for a $D$-dimensional system is [3]

$$
f_{i}^{L E}(\rho, \mathbf{u})=\frac{\rho}{b}+\frac{\rho D}{b c^{2}}\left(\mathbf{c}_{i} \cdot \mathbf{u}\right)+\frac{\rho}{b} G(\rho)\left[\left(\mathbf{c}_{i} \cdot \mathbf{u}\right)^{2}-\frac{c^{2} u^{2}}{D}\right]+O\left(u^{3}\right)
$$

with

$$
G(\rho)=\frac{D^{2}}{2 c^{4}} \frac{b-2 \rho}{b-\rho}
$$

and

$$
c^{2}=\frac{1}{b} \sum_{i=1}^{b} c_{i} \cdot c_{i}
$$

$c$ is related to the speed of sound $c$, via

$$
c_{s}^{2}=\frac{1}{D} c^{2}
$$

One can obtain the familiar hydrodynamic equations by assuming weak space and time dependence of $\rho$ and $\mathbf{u}$. If so, one can expand the one-particle non-equilibrium distribution around the local-equilibrium distribution function. In continuous systems this is know as the Chapman-Enskog expansion; in the same spirit a multi-scale expansion is used for lattice gases [3]. The basic result is that, provided that the lattice has sufficiently high symmetry, the final macroscopic equations become equivalent to the Navier-Stokes equation for an incompressible fluid as long as the flow velocity is much less than the speed of sound, and all spatial variations in the system occur on a scale that is large compared with the mean free path of the lattice gas particles. In this repect LGCA model atomic fluids. A final remark is that, as the total momentum in the system $\mathbf{P}$, and the total number of particles $N$, is conserved, the averaging is performed in the $\{N, \mathbf{P}\}$ ensemble. We define the mean occupation number $d$ as

$$
d=\frac{N}{b V}
$$

where $V$ is the number of lattice nodes in the system and $b$ is the number of possible velocities at one node. Clearly $d$ represents the probability that a single link is occupied. If the system is in equilibrium and the total momentum $P$ vanishes, $d$ should be uniform over all directions and sites.

\section{Velocity autocorrelation function of a tagged particle}

\subsection{Introduction}

The velocity autocorrelation function (VACF) of a tagged particle is a function of fundamental importance in kinetic theory. If the tagged particle has velocity $\mathrm{v}(t)$ at 
time $t$, the VACF is generally defined as the time average of the product $v_{x}(0) v_{x}(t)$ along any possible trajectory of the tagged particle, viz.

$$
\left\langle v_{x}(0) v_{x}(t)\right\rangle=\lim _{T \rightarrow \infty} \frac{1}{T} \int_{0}^{T} d s v_{x}(s) v_{x}(t+s)
$$

In a Molecular Dynamics simulation, a single particle has a well defined trajectory, and (5) can be applied directly. As time is restricted in a simulation, $T$ will be finite, and there will be a certain statistical error in the $\left\langle v_{x}(0) v_{x}(t)\right\rangle$, which is proportional to $T^{-1 / 2}$ and independent of $t$, for $t$ larger than the initial decay time [5]. As fluctuations decay, the VACF decreases for 'long' times. As a consequence, the signal-to-noise ratio is decreasing as well. This makes it difficult to study the long-time behavior of the VACF using Molecular Dynamics, and will require lengthy simulations (large $T$ ). In a lattice gas simulation the particles are in principle indistinguishable, as they will loose their identity in each collision. Hence a tagged particle is ill-defined. A straightforward way to get around this problem is to apply stochastic rules at each collision with a tagged particle involved, defining which one of the post-collisional particles is the tagged particle. This can be done, for instance, by random selection. In this way, (5) can be applied again, but also in this case there is brute force required to observe the VACF for longer times $[6,7]$. In order to circumvent this problem, we developed a method with which the VACF can be calculated much more efficiently. This method is described below.

\subsection{Moment-Propagation method}

In general, the statistical accuracy of the VACF can be improved by averaging over different trajectories, instead of just one, as is done in eqn.(5). The idea of the moment propagation method $[8,9]$ is that in a lattice-gas simulation it is possible to simultaneously follow all possible trajectories of the tagged particle. The stochastic rules define the probability $P_{n}$ of each trajectory $n$. In this way, we construct the VACF as

$$
\left\langle v_{x}(0) v_{m}(t)\right\rangle_{t r}=\sum_{n} P_{n} v_{x}(0) v_{x}^{n}(t)
$$

where $v_{x}^{n}(t)$ is the velocity of the tagged particle after time $t$, if it would have followed trajectory $n .\langle\cdot\rangle_{t r}$ stands for the average over all trajectories. Keeping track of all the trajectories is very time and memory consuming, but also not necessary. In lattice gases, particles that have followed trajectories $n$ that arrive at time $t$ at the same site $r$ and link $i$ will have the same final velocity $v_{x}^{n}(t)=c_{i x}$. Therefore, in equation (6) we can group trajectories that arrive at the same coordinates $(\mathbf{r}, i)$ at time $t$ :

$$
\sum_{n} P_{n} v_{m}(0)\left(v_{x}(t)\right)_{n}=\sum_{r, i} v_{x}(0) c_{i x} \sum_{n(r, i)} P_{n}
$$

where the sum over $n(r, i)$ is over all trajectories ending at $(r, i)$. Clearly

$$
\sum_{n(\mathbf{r}, i)} P_{n}=P(\mathrm{r}, i ; t)
$$

represents the total probability (= via any possible trajectory) of the tagged particle to arrive at $(\mathbf{r}, i)$ after time $t$. If the stochastic rules are such that in a 'tagged-particle collision' one of the post-collision particles is tagged at random, $P(\mathbf{r}, i ; t)$ can be written 
as the product of the probability $P(\mathbf{r} ; t)$ of the tagged particle to arrive site $\mathbf{r}$ before the collision, and the probabilty that the tagged particle will be scattered into the $i$-th direction after the collision, viz.

$$
P(\mathbf{r}, i ; t)=P(\mathbf{r} ; t) \frac{s_{i}(\mathbf{r}, t)}{\rho(\mathbf{r}, t)}
$$

$s_{i}$ and $\rho$ refer to the state just after the collision. Note that the total number of particles $\rho(\mathrm{r}, t)$ is not changed in a collision. If we substitute this into equation $(7,8)$, and do the summation over $i$, we get we get the very simple expression

$$
\left\langle v_{x}(0) v_{x}(t)\right\rangle_{t r}=\sum_{\mathbf{r}} P(\mathbf{r} ; t) v_{x}(0) u_{x}(\mathbf{r}, t)
$$

The probability $P(\mathrm{r} ; t)$ can be constructed from the probability at the neighboring sites one time step earlier

$$
P(\mathbf{r}, t)=\sum_{i}\left[P\left(\mathbf{r}-\mathbf{c}_{\boldsymbol{i}}, t-1\right) \frac{s_{i}\left(\mathbf{r}-\mathbf{c}_{\boldsymbol{i}}, t-1\right)}{\sum_{j} s_{j}\left(\mathbf{r}-\mathbf{c}_{\boldsymbol{i}}, t-1\right)}\right]
$$

where all the states $s$ are after collision states. If the particle starts at $\left(\mathbf{r}^{\prime}, i^{\prime}\right)$, at time $t=0$, the value $P(\mathbf{r}, 1)$ is given by

$$
P(\mathbf{r}, 1)=\delta\left(\mathbf{r}-\mathbf{c}_{\mathbf{i}^{\prime}}, \mathbf{r}^{\prime}\right)
$$

This also implies that $v_{x}(0)$ is equal to $c_{i^{\prime} x}$. Equation (10),(11) and (12) form a scheme that can be applied directly in a lattice gas simulation. The above scheme is even more efficient if we use all particles in the lattice gas as a starting particle. As everything in the above scheme is linear, we can just add all contributions from different tagged particles in $P(\mathbf{r}, t)$, which now represents the sum of the 'arriving probability' of all tagged particles. The only thing to be careful about is when we start correlating, as different particles have different initial velocities. This is taken into account if we weight the initial value $P(\mathbf{r}, 1)$ by the initial tagged particle velocities, and subsequently leave the initial velocity out of equation (10) :

$$
P(\mathbf{r}, \mathbf{1})=s_{i}\left(\mathbf{r}-\mathbf{c}_{i}, 0\right) c_{i x}
$$

The evolution of $P$ is still given by equation (11), and the VACF at time $t$ is then

$$
\left\langle v_{x}(0) v_{x}(t)\right\rangle_{t r}=\frac{1}{N} \sum_{\mathbf{r}} P(\mathbf{r} ; t) u_{x}(\mathbf{r}, t)
$$

where $N$ is the total number of particles. Equation (11),(13) and (14) are the basic equations of the MP-method. As the essence of the method is the propagation of the first moment of the single particle distribution function, we call it the momentpropagation method. In general we can propagate any moment of the distribution function. Note that the VACF is only averaged over all possible trajectories of all starting particles of one particular initial configuration. The big advantage of this method is that the number of possible trajectories increases with time, hence the statistical accuracy increases. Simulations in two- and three dimensions show that the statistical error decreases sligthly slower than $t^{-1}$. For longer times, the VACF itself decays as $t^{-1}$ and $t^{-1.5}$ in $2 \mathrm{D}$ and $3 \mathrm{D}$ respectively, so the signal-to-noise ratio is only slowly decreasing in time. To increase the overall accuracy, additional timeor ensemble averages can be done, which is also useful to estimate the error in the 'trajectory averaged' function. 


\subsection{VACF after one timestep}

With the above formalism, it is easy to show how to obtain the exact result for the VACF after one time step. Suppose the tagged particle starts at $t=0$ from site $\mathbf{r}-\mathbf{c}_{i}$ into direction $i=1$. According to equation (10) the 'trajectory-averaged' VACF is

$$
\left\langle v_{x}(0) v_{x}(1)\right\rangle_{t r}=c_{1 x} u_{x}(\mathbf{r}, 1)
$$

as the probability $P(\mathbf{r} ; 1)$ of the tagged particle to arrive at site $\mathbf{r}$ at time $t=1$ is equal to one, and zero for all other sites. This is for one configuration. We can perform the exact averaging over all members of the ensemble by averaging (15) over all possible configurations of the other links at $\mathbf{r}$ before the collision. As we now sum over all states at one particular site $\mathbf{r}$ at one particular time $t=1$, it is convenient to drop space and time variables, and have $\rho$ and $u_{x}$ directly dependent on the one-node state $s$. Note that in formula (15) $u_{\text {w }}$ refers to the after-collision state. As momentum is conserved in a collision, this can refer as well to the pre-collision state. So we can write

$$
\left\langle v_{x}(0) v_{x}(1)\right\rangle=\sum_{d} P(s) \frac{\delta\left(s_{1}, 1\right)}{d} c_{1 x} u_{x}(s)
$$

where the sum is over all possible one-node pre-collision states $s . P(s)$ is the probability that the one-node state $s$ occurs in the given ensemble. The term $\delta / d$ corrects for the fact that we should not go over all states, but only over states for which a particle is present in direction 1, as we know for sure that the tagged particle arrived there before the collision. The probabilities for different links to be occupied are completely independent, so we can write

$$
P(s)=d^{p(s)}(1-d)^{b-\rho(s)}
$$

if the total momentum in the system is vanishing. Note that, as we sum over all precollision states, $\left\langle v_{x}(0) v_{x}(1)\right\rangle$ is independent of the specific collision rules. Finally, we should also average over all possible starting directions of the tagged particle. The final expression becomes

$$
\left\langle v_{x}(0) v_{x}(1)\right\rangle=\frac{1}{b} \sum_{i=1}^{b} \sum_{a} d^{p(s)-1}(1-d)^{b-p(s)} \delta\left(s_{i}, 1\right) c_{i x} u_{x}(s)
$$

With the above scheme $\left\langle v_{x}(0) v_{x}(1)\right\rangle$ can be obtained by brute force, that is, by counting $b$-times over all $2^{b}$ states, which can be done by hand or on a computer. This has been done in [18] for the three-dimensional FCHC-model. However, it is possible, and more elegant, to derive from (18) a compact expression for $\left\langle v_{x}(0) v_{x}(1)\right\rangle$. This has been done by Ernst and Naitoh [10]. Their final expression is, for systems with single species particles, without rest particles:

$$
\left\langle v_{x}(0) v_{x}(1)\right\rangle=\left\langle v_{x}^{2}\right\rangle \frac{1-d}{d(b-1)}\left[1-(1-d)^{b-1}\right]
$$

To be complete, we also give the Boltzmann diffusion coefficient $D_{0}$ that can be evaluated directly from $\left\langle v_{x}(0) v_{x}(1)\right\rangle$. If we introduce the normalized VACF $Z_{N}(1)=$ $\left\langle v_{x}(0) v_{x}(1)\right\rangle /\left\langle v_{x}^{2}\right\rangle$, we can evaluate $D_{0}$ by the discrete equivalent of the Green-Kubo integration [11]:

$$
D_{0}=\left\langle v_{x}^{2}\right\rangle\left[\frac{1}{2}+\sum_{k=1}^{\infty}\left\langle Z_{n}(1)\right\rangle^{k}\right]
$$




$$
\left.=\left\langle v_{\boldsymbol{x}}^{\mathbf{2}}\right\rangle\left[\frac{1}{2}+\frac{Z_{N}(1)}{1-Z_{N}(1)}\right)\right]
$$

Finally, the mean free time $t_{0}$ is given by

$$
t_{0}=-\left(\ln Z_{N}(1)\right)^{-1}
$$

\subsection{Finite size corrections}

The VACF measured as described above, is obtained in the $\{N, \mathbf{P}\}$ ensemble, or Molecular Dynamics(MD-) ensemble. In theoretical calculations normally the canonical ensemble is used, in which the total momentum of each state fluctuates around an average $\langle\mathrm{P}\rangle=0$. If the total momentum in the system is exactly zero, this means that if the tagged particle has a velocity $v$, the fluid particles have, on average, a small veloc-

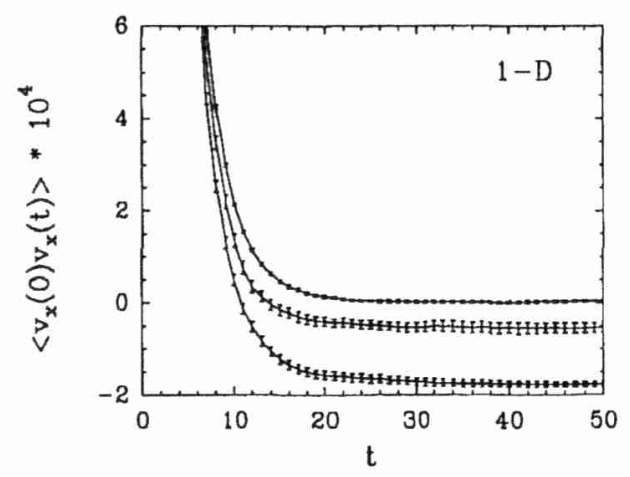

Figure 1. Normalized velocity autocorrelation function of a tagged particle in a onedimensional lattice gas model, for system size $L=500$ (lower curve), $L=2000$ (middle curve) and $L=100000$ (upper curve). Note the negative plateaus for the two small systems; these plateaus represent the anti-correlation part (22) of the VACF. The density (defined by (4)) is equal to 0.7 .

ity $-v / N$ [14]. This will give an anticorrelation part in the VACF which is equal to $\left\langle v_{x}^{2}\right\rangle(1-d) / N$, and so the simulation result in the LGCA will be dependent on the number of particles. This anti-correlation part is seen most clearly in the VACF of a tagged particle in a one-dimensional model. As all other velocity correlations decays to zero very quickly, this anti-correlation part completely dominates the behavior and the measured VACF will have a negative plateau. This is clearly observed in figure 1. In the simulations we used the one-dimensional model introduced by d'Humières et al. [12]. In this model, particles can reach nearest-neighbor and next-nearest-neighbor sites in one timestep. Also particles can stay at their site. Hence the number of velocities $b$ is equal to 5 . In figure 1 , we plotted the one-dimensional normalized 
correlation function for different system sizes. The lowest, middle and upper curve correspond to systems of 500,2000 and 100000 sites respectively. The density was set to $d=0.7$, so the number of particles $N$ in these systems was equal to 1750,7000 and 350000 , respectively. The values of the negative plateaus observed in figure 1 turn out to be equal to $(1-d) / N$. In the more systematic study by Naitoh et al. [13] it was shown that, in order to improve the agreement of the simulation results for different system sizes with those for the infinite system, the measured VACF must be corrected by this factor :

$$
\left\langle v_{x}(0) v_{x}(t)\right\rangle=\left\langle v_{x}(0) v_{x}(t)\right\rangle_{N P}+\frac{\left\langle v_{x}^{2}\right\rangle(1-d)}{N}
$$

This is a correction that should be made on all simulation data. When compared with specific theories, for instance mode-coupling theories, one should still take into account the finite volume of the system. In that case, one should for instance consider finite- $k$ sums. The comparison of the measured VACF with mode-coupling theories in small systems is studied extensively in [13].

\subsection{Examples}

In this section we show examples of the VACF measured with the moment-propagation method, in several well-known models. At this point it is convenient first to spent a few words on the behavior of the VACF in general. For short times, one expects that the decay of the VACF is close to exponential decay. This is because correlations between colliding particles are not yet dominant and the Boltzmann approximation holds. However, for longer times, these correlations will dominate the behavior and will manifest themselves in a slower decay of the VACF. The latter behavior was first observed by Alder and Wainwright in a seminal Molecular Dynamics simulation [14]. The Alder-Wainwright results showed that in the long-time limit the functional form of the VACF was compatible with an algebraic decay $\sim t^{-D / 2}$, where $D$ is the dimension of the system. This behavior was explained by Alder and Wainwright using a dimensional argument, and subsequently also from linear hydrodynamics in the modecoupling theory by Ernst et al [15], and from kinetic theory by Dorfman and Cohen [16]. The mode-coupling theory has been extended to lattice gases by Ernst $[17,18]$, so we can do a quantitative comparison between the lattice gas simulation results and the mode-coupling theory. In figures $2 \mathrm{a}-\mathrm{c}$ we have plotted the simulation results and the theoretical predicitons for respectively two,three and four dimensions. The specific models used are respectively the (2D)FHP-III model, the projected (3D)FCHC model and the full (4D)FCHC model. In these figures we plotted the two extreme theoretical predictions : The dashed line represents the exponential decay that follows from the Boltzmann assumption (no correlations), and is valid for very short times. The solid line represent the predicition for the VACF in the long-time limit as predicted from mode-coupling theory [17]. Up to now there has been no full theory to account for the intermediate behavior. As we can see from figure $2 \mathrm{a}-\mathrm{c}$, we find good agreement between the measured correlation function (squares) and the corresponding theories, in both time regimes. Note that in all cases the error (crosses) is decreasing in time, about as 


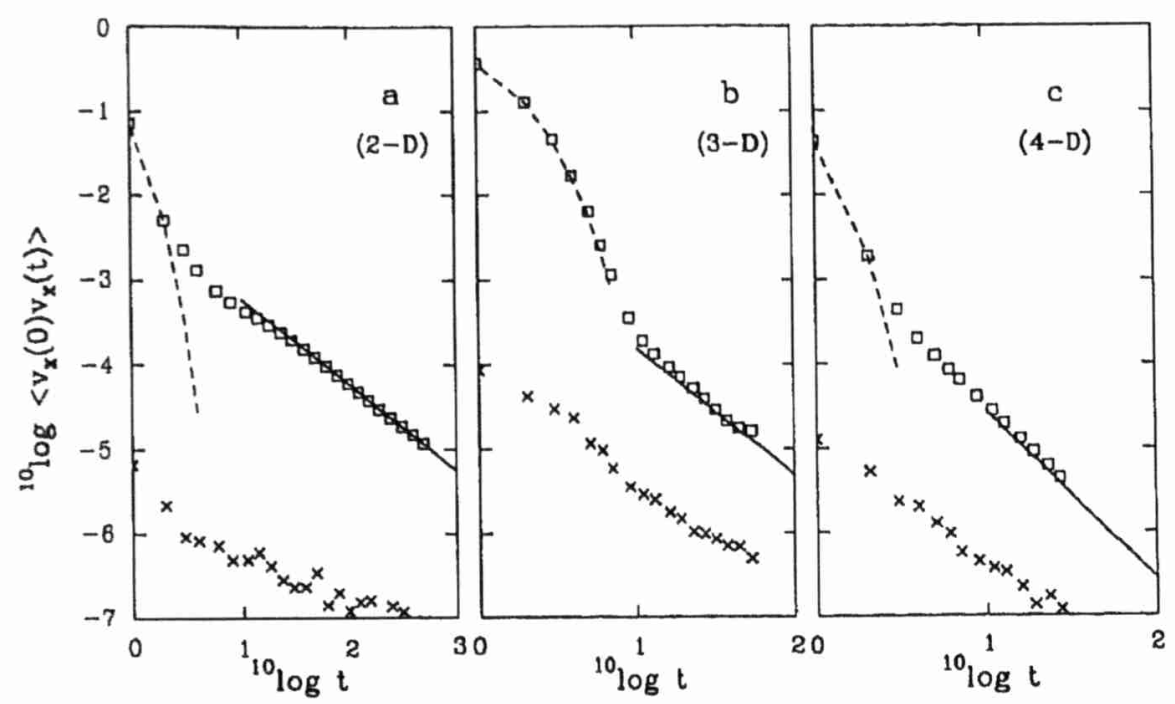

Figure 2a-c. Normalized velocity autocorrelation function of a tagged particle in a twothree- and four-dimensional lattice gas fluid. The squares are the data obtained from a simulation using the moment propagation method. The crosses represent the statistical error. Note that the error is decreasing in time. The dashed lines represent the theoretical predictions in the Boltzmann assumption. The solid lines are the predicitions from mode-coupling theory for the long-time decay of the VACF. The specific models are (a) two-dimensional FHP-III model, at density $d=0.7$, (b) three-dimensional FCHC model, at density $d=0.1$, (c) four-dimensional FCHC model, at density $d=0.5$.

fast as the correlation function itself, so we have almost constant signal-to-noise ratio. More extensive reports on these simulations and the comparison with mode-coupling theory can be found in $[13,18,19,20,21]$.

\subsection{Final remarks}

The moment-propagation method has proven to be a very powerful tool to calculate the VACF of a tagged particle. Unfortunately, it cannot be used to calculate the stress-stress correlation function. In the MP-method it is essential to average over different paths of a tagged particle. This limits the method to correlation functions of single particle properties. For the stress-stress correlation function one should correlate $\left[\sum_{i, r} s_{i}(\mathrm{r}, t) c_{i x} c_{i y}\right]$, which is a collective property. This means one always get the same outcome for the function if one uses the same initial conditions, irrespective of what trajectories the individual particles have followed; so averaging over different trajectories will give no improvement in statistics. However, there is a wide range of other applications of the MP-method. One of them is to calculate the VACF in lattice-Lorentz systems $[8,22]$. A completely different application can be found in polymer physics. There the method can be used to efficiently grow polymers on a lattice $[23,24,25]$. 


\section{Colloidal systems}

\subsection{Introduction}

In simple liquids there is a large space- and time scale separation between microscopic processes and macroscopic processes. By introducing colloidal particles in the liquid one introduces an intermediate 'mesoscopic' space and time scale. In fact, there are several distinct timescales that are relevant for the description of colloidal dynamics. In particular, these times are the time it takes particles to interact via the hydrodynamic modes, the relaxation time of the momentum of the colloidal particles, and the time at which the configuration of colloidal particles changes significantly, i.e. the time it takes a particle to displace a substantial fraction of its own diameter. Theoretically one can make some headway by assuming that all these timescales are well separated; so one would like to assume that the hydrodynamic interactions are 'instantaneous' compared to the typical velocity relaxation time of the colloidal particles, while on the same timescale, one would like to consider the spatial configuration of the colloidal particles as 'frozen'.

Colloidal suspension are expensive to simulate because very long simulations of very large systems are needed to capture all relevant timescales. In simulations of simple liquids it was demonstrated that the separation between microscopic and macroscopic scales does not need to be of the order of $10^{8}$. In fact, this was already demonstrated by the observation of the long-time tail by Alder and Wainwright in a molecular dynamics simulation [14]. This long-time tail can be explained in terms of macroscopic hydrodynamic arguments. Yet the long-time tail could already be observed in a system of at most a 1000 particles, at times of the order of 10 mean collision times. In a colloidal suspension simulation on cannot cut down the separation between microscopic and macroscopic by this amount, as all the intermediate scales have to fit in between. The approach of the 'Brownian dynamic' simulations, initiated by Ermak and McCammon [26], is not to start at the microscopic level, but at the level of Langevin equation. The timestep at which the system is updated is then much larger than the velocity relaxation time of the colloidal particles, but much smaller than the time it takes the configuration to change significantly. Hence a good timescale separation is implicit in this method. The thermal fluctuations are incorporated artifically, assuming that these fluctuations are a Gaussian process. The Langevin description leads to pure diffusive behavior of the particles, and therefore ignores the persistence of velocity of the Brownian particles due to the vortex recirculation. A similar approach is followed in the 'Stokesian' dynamics simulation by Brady and Bossis [27]. However, the latter technique does account for the hydrodynamic interactions between the colloidal particles. Ladd, Colvin and Frenkel [2] introduced a model that essentially starts at the microscopic level. In this model, the fluid is represented by a lattice gas cellular automaton. As was discussed in section 2, such models have the correct hydrodynamic behavior. Moreover, thermal fluctuations and correlations are also intrinsically present, as is shown is the results of tagged particle diffusion in section 3. The advantage of these lattice gas models over molecular dynamics models, is that they are computationally much faster. With the approach of ref. [2] it should in principle be possible to cover all the timescales from the microscopic time on. As a consequence, this model allows one 
to test whether microscopic correlations, which are ignored in the Brownian dynamic simulations, have an influence on the mesoscopic behavior of the colloidal particle. In addition, the timescale separation assumed in the theories can be tested. The vadility of the assumption underlying this separation was put into question by Masters [28].

It is a non-trivial problem to incorporate a large colloidal particle in a lattice gas. Such a hybrid approach requires a correct interaction at the microscopic level between the discrete lattice gas particles and the 'continuous' moving surface of the colloidal particles. One efficient method to incorporate a moving boundary will be described in the section below.

\subsection{Moving boundary rules}

One essential ingredient of the moving-boundary rules is that these rules result, on average, in a dissipative force on the suspended particle. An obvious choice of rules is those according to which the fluid next to the solid particle moves with the local velocity of the surface of the solid particle. In this way one models the hydrodynamic 'stick' boundary condition; in an infinite three dimensional system this which will give rise to a frictional force on the particle $F=6 \pi \eta R \mathrm{U}$, where $R$ is the hydrodynamic radius of the particle, $\eta$ is the shear viscosity and $U$ is the velocity of the suspended particle. Our specific method originates from an idea by Rem and Somers [29] and was subsequently worked out and implemented by Ladd and Frenkel [30,31]. We only give a simple outline of the idea, a more complete and rigorous account has been given by Ladd [32]. The rules are applied locally at the sites of the lattice closest to the surface (boundary) of the particle. In case of a zero-velocity boundary, one can model stickboundary conditions very easily by having the fluid particles at these sites 'bounce back'. This means that if a particle approaches the boundary with velocity c, it will return with velocity -c. If one defines the local fluid velocity as the average of the fluid velocity before and after this rule is applied, this implies a zero local fluid velocity in all cases. The basic idea of the method is to have corrections to this rule if the boundary has a non-zero velocity. This correction consists of letting particles go straight into the direction of the local boundary velocity with some probability, instead of bouncing back. Thus this probability will depent on the local boundary velocity. As the wall can have velocitics in both directions, these rules immediately imply that there should be fluid particles inside the particle as well as outside. The change in momentum of the fluid due to these rules will be converted into forces and torques on the solid particle. In this way the rules represent collisions of the lattice gas particles with the wall of the solid particles, in which momentum is conserved. Note that there can be no collision rules in which both momentum and energy are conserved, because the lattice gas particles can only have discrete velocitics. We will describe the rules now in a little more detail. First one selects pairs of links $\{i,-i\}$ that point in opposite direction, so $c_{i}=-c_{-i}$. We have the state of such a pair given by their occupation numbers $\left(s_{i}, s_{-i}\right)$. We calculate the local boundary velocity $\mathrm{v}$ from the the linear velocity $U$ and the angular velocity $\omega$ of he solid particle

$$
\mathbf{v}=\mathbf{U}+\omega \times \mathbf{r}
$$


where is $\mathbf{r}$ the distance from the center of the particle. We define our link pair $\{i,-i\}$ such that $\mathbf{v}$ has a positive component in the direction of $i$, viz.

$$
x_{i}=\mathrm{c}_{i} \cdot \mathrm{v}>0
$$

The rules are now such that the state $\left(s_{i}, s_{-i}\right)=(0,1)$ will always bounce back; on the other hand, the state $\left(s_{i}, s_{-i}\right)=(1,0)$ will bounce back with a probability $1-p_{i}$, and will remain unchanged with probability a $p_{i}$. Clearly for the states $(1,1)$ and $(0,0)$ both rules have the same effect. So we have the transition probabilities given by

$$
\begin{aligned}
& (0,1) \stackrel{1}{\longrightarrow}(1,0) \\
& (1,0) \stackrel{1-p_{i}}{\longrightarrow}(0,1) \\
& (1,0) \stackrel{p_{i}}{\longrightarrow}(1,0)
\end{aligned}
$$

We next define $g_{10}$ and $g_{01}$ as the probabilities to have state $(1,0)$ and $(0,1)$ respectively, before the rules are applied. The new probabilities $g_{10}^{\prime}$ and $g_{01}^{\prime}$ after the rules are applied are then given by

$$
\begin{aligned}
& g_{10}^{\prime}=g_{01}+p_{i} g_{10} \\
& g_{01}^{\prime}=\left(1-p_{i}\right) g_{10}
\end{aligned}
$$

If the system is stationary we must have $g_{10}=g_{10}^{\prime}$ and $g_{01}=g_{01}^{\prime}$. This implies that

$$
g_{01}=\left(1-p_{i}\right) g_{10}
$$

We can write the two-particle distribution function $g$ as the product of the one-particle distribution function $f$ introduced in section 2 :

$$
\begin{aligned}
& g_{10}=f_{i}\left(1-f_{-i}\right) \\
& g_{01}=f_{-i}\left(1-f_{i}\right)
\end{aligned}
$$

For $f$ we use the small-velocity expansion of the local-equilibrium distribution function (3)

$$
f_{i}=\frac{\rho}{b}+\frac{\rho D}{b c^{2}} x_{i}+\frac{\rho}{b} G(\rho)\left(x_{i}^{2}-\frac{c^{2} v^{2}}{D}\right)+O\left(v^{3}\right)
$$

where $G(\rho)$ is defined in (3). In most of our simulations we use a density $\rho=b / 2$, which corresponds to a $50 \%$ filling of the lattice. At this particular density the convective term drops out of the Navier-Stokes equation, so the dynamics of the system can be described by the so-called 'creeping-flow' fluid equations. As $G(b / 2)=0$, the third term of the expansion (26) vanishes. Combining equations (24),(25) and (26) yields

$$
\frac{1}{4}-\frac{D}{2 c^{2}} x_{i}+\frac{D^{2}}{2 c^{4}} x_{i}^{2}=\left(1-p_{i}\right)\left[\frac{1}{4}+\frac{D}{2 c^{2}} x_{i}+\frac{D^{2}}{2 c^{4}} x_{i}^{2}\right]
$$

where we made use of $x_{-i}=-x_{i}$. The solution is

$$
p_{i}=\frac{c^{2}}{D} \frac{4 x_{i}}{\left(x_{i}+\frac{c^{2}}{D}\right)^{2}} .
$$

The procedure in a simulation is now as follows. One performs the normal collisions at all sites of the lattice, except at the boundary sites. At the same time one goes over all these boundary sites and determines the local boundary velocity $v$ for each site. At 
one site, one goes over all link pairs, determines $p_{i}(v)$ and updates the state according to (23). The change in momentum of the lattice-gas particles due to these rules are then turned into forces and torques on the colloidal particle which are then used to update the particle velocities and angular velocities, according to the assigned mass $M$ and moment of inertia of the solid particle. In the study by Ladd and Frenkel [31] it was found that the dissipative hydrodynamic forces arising from these rules, did agree within a few percent with the essentially exact solutions of the creeping flow equations for the same system (a periodic array of spheres). Also the drag force between two spheres moving in opposite direction was in good agreement with the exact solutions, even when the surfaces of the two particles are close to touching. In the next section we will give results obtained with the above model for a single suspended particle in a two-dimensional system.

\subsection{Diffusion in two dimensions}

In our simulations we used a two-dimensional triangular lattice gas, with a lozengeshaped unit cell; the size of the lattice was $256 \times 256$ sites. We inserted a single colloidal particle with a radius of 2.5 lattice spacings; The mass of this particle was equal to 680. The lattice gas model used was a FHP-I model, which differs slightly from Ref. [1] in that all possible mass and momentum conserving collisions are included. The reduced fluid density was set to $\rho=\sqrt{12}$, corresponding to a $50 \%$ filling of the lattice. At this particular fluid density the long-time tail in the stress-stress correlation function vanishes [33]; hence the kinematic viscosity is time independent, well defined, and equal to the Boltzmann viscosity $\nu_{0}=0.09722$. For reasons to be explained below, we artificially constrained the particle to its initial position, even though it had a nonzero velocity. We measured the VACF of the colloidal particle every timestep up to 1000 timesteps, and the virtual displacements (by integrating the velocity) every 100 timesteps up to 5000 timesteps. The mean values were calculated by time averaging (see eqn.(5)) over 250 million time steps, after having equilibrated the system for one million timesteps.

Before we show the results we briefly adress to the problem of diffusion in two dimensions. The explicit prediction for the long-time tail in the VACF (see also section 3 ) in a two-dimensionsal fluid is $[14,15,16]$ :

$$
\left\langle v_{x}(0) v_{x}(t)\right\rangle=\frac{b}{(D+\nu) t} .
$$

In this equation, $b=M^{*}\left\langle v_{x}^{2}\right\rangle / 8 \pi \rho$, where $\rho$ is the mass density of the fluid, and $M^{*}$ is the effective mass of the diffusing particle. $D$ is the self-diftusion coefficient of the colloidal particle, defined as

$$
\begin{gathered}
D=\lim _{t \rightarrow \infty} \frac{1}{4 t}\left\langle(\mathbf{r}(t)-\mathbf{r}(0))^{2}\right\rangle \\
=\lim _{t \rightarrow \infty} \frac{1}{4 t} \int_{0}^{t} d t^{\prime} \int_{0}^{t^{\prime}} d s\left\langle v_{x}(0) v_{x}(s)\right\rangle .
\end{gathered}
$$

$\nu$ the kinematic viscosity of the suspension. Let us assume for simplicity that $\nu$ is independent of time, a condition that is indeed satisfied in our simulation. From equation (29) it follows immediately that equation (28) is inconsistent: if the VACF decays 
as $t^{-1}$, then the integral $D$ diverges as $\ln t$; in that case, according to (28), the decay cannot longer be $t^{-1}$. As was first suggested by Wainwright et al. [35], a self-consistent picture can be obtained by introducing time-dependent transport coefficients $D(t)$ and $\nu(t)$. In this case the diffusion coefficient diverges as $D(t)=\sqrt{2 b \ln t}$; the corresponding VACF is proportional to $(t \sqrt{\ln t})^{-1}$. So we see that in two dimensions one expects a renormalisation of the diffusion constant. The reason why we introduced a colloidal particle constrained to its initial position is that this forces $D(t)$, as it appears in (28), to be equal to zero. This decouples eqn.(28) and (29), so there is no need for renormalisation. The long-time tail is then given simply by $(b / \nu) t^{-1}$. The 'virtual' diffusion coefficient, which can be measured by means of (29), as the particle has a non-zero velocity, diverges as $(b / \nu) \ln t$. Both these effects are observed in our simulations, as can be seen in figure 3 .
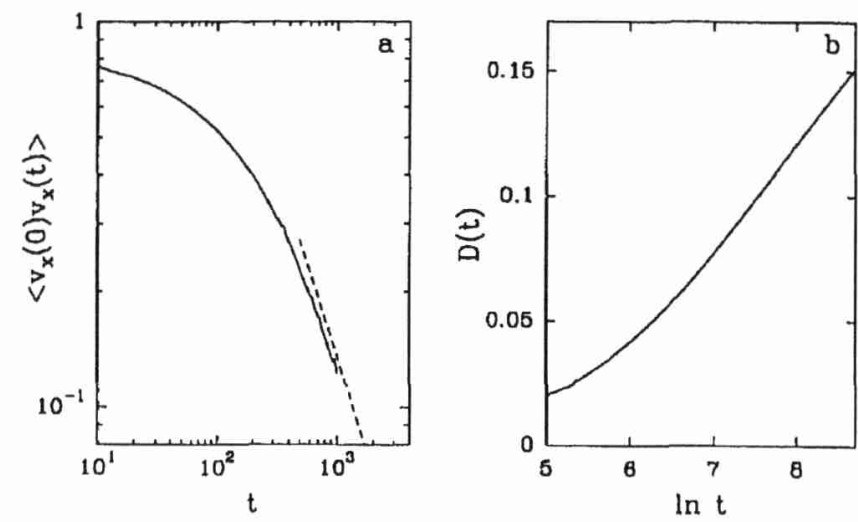

Figure 3. (a) Normalized velocity autocorrelation function of a single colloidal particle in a two-dimensional lattice gas fluid, vs. time, on a log-log scale. The solid line represents the data obtained from the simulation. Note that the function approaches a power-law decay consistent with $t^{-1}$. The dashed line is a line with slope-1 drawn for comparison. The error in our data points is on average $1.310^{-3}$.

(b) Simulation result for the time dependent diffusion coefficient vs. the natural logarithm of time, in the same system. The diffusion coefficient is defined by the meansquare displacement devided by time. Note that the diffusion coefficient becomes linear in $\ln t$, which corresponds to a strict $t^{-1}$ decay of the velocity autocorrelation function.

In figure 3n, we show a $\log -\log$ plot of the velocity autocorrelation function vs. time. The solid line is the data obtained in the simulation. We indeed observe that a power law decay $\sim t^{-1}$ is approached. The dashed line is a line with slope -1 drawn for comparison. In figure $3 \mathrm{~b}$, we show the virtual diffusion coefficient $D(t)$ measured in the simulation vs. the logarithm of time. The straigth line indicates that $D(t)$ indeed diverges as $\ln t$. A more extensive report on these simulations can be found in [34], where we report also the observation of the renormalisation of $D(t)$ for a 'normal' unconstrained particle. We note that the renormalisation effects are very small in the tagged particle diffusion of section 3 , and it was therefore ignored in the discussion. Nevertheless, extensive simulations in a two-dimensional lattice gas have demonstrated the existence of the effect. 


\subsection{Perspectives}

As we have shown above, the lattice-gas approach for simulating colloidal systems looks promising. The model seems to be able to account for much of underlying physics of these complicated systems, even more subtle aspects like renormalisation of diffusion in two dimensions. Yet, the present model is not completely satisfactory in all aspects. In particular, one would like to have energy conserved in this hybrid-model, but there is no straightforward way to include this. A better understanding of this is clearly desirable. One of the most important applications of the lattice gas-colloidal model is eventually to simulate dense suspensions of many particles. At present simulations with many hundreds of spheres are possible on present-day supercomputers, or dedicated hardware [36]. Moreover, one is not restricted to the relatively simple sytems of spherical particles. Complicated systems with arbitrary shaped particles or polymers, even in complex geometries, can be studied with only little extra effort. In these systems, precise theoretical predictions are extremely difficult to make, hence the lattice-gas colloidal-particle model can really help to gain a better understanding of these systems.

\section{Acknowledgements}

This work has been carried out in close cooperation with Tony Ladd and Matthieu Ernst. Much of the work that we have performed has been inspired by the seminal work of Bernie Alder. We gratefully acknowledge his continuing interest in this work. The work of the FOM-Institute is part of the scientific program of FOM and is supported by the Nederlandse Organisatie voor Wetenschappelijk Onderzoek (NWO).

\section{References}

[1] U. Frisch, B. Hasslacher and Y. Pomeau, Phys. Rev. Lett. 56, 1505(1986).

[2] A.J.C. Ladd, M.E. Colvin and D. Frenkel, Phys. Rev, Lett. 60, 975(1988).

[3] U. Frisch, D. d'Humières, B. Hasslacher, P. Lallemand, Y. Pomeau and J.-P. Rivet, Complex Systems 1, 649(1987).

[4] D. d'Humières and P. Lallemand, Complex Systems 1, 599(1987).

[5] R. Zwanzig and N.K. Ailawadi, Phys. Rev. 182, 280(1969).

[6] A. Noullez and J.-P. Boon, Physica D 47, 212(1991).

[7] P.M. Binder and D. d'Humières, in Proceedings of the workshop on Discrete kinetic theory, lattice gas dynamics and foundations of hydrodynamics, Torino, Italy, Sept.20-24, 1989, edited by R. Monaco (World Scientific, Singapore, 1989).

[8] D. Frenkel in : Proceedings of workshop on Cellular Automata and Modelling of Complex Physical Systems, Les Houches, France, Feb. 21-28, 1989, edited by P. Manneville (Springer, Berlin, 1989).

[9] D. Frenkel and M.H. Ernst, Phys. Rev. Lett. 63, 2165(1989).

[10] M. H. Ernst and T. Naitoh, J. Phys. A: Math. Gen. 24, 2555(1991). 
[11] M. H. Ernst and J.W. Dufty, J. Stat. Phys. 58, 57(1990); J.W. Dufty and M.H. Ernst, J. Phys. Chem. 93, 7015(1989).

[12] D. d'Humières, P. Lallemand and Y.H. Qian, C.R. Acad. Sci. Paris II 308, 585(1988); Y.H. Qian, Gaz sur Réseaux et Théorie Cinétique sur Réseaux Appliquée à l'Equation de Navier-Stokes, Ph.D. thesis, Univ. P. et M. Curie, Jan. 1990.

[13] T. Naitoh, M.H. Ernst, M.A. van der Hoef and D. Frenkel, Phys. Rev. A43(1991) in press.

[14] B.J. Alder and T.E. Wainwright, Phys. Rev. A1, 18(1970).

[15] M.H. Ernst, E.H. Hauge and J.M.J. van Leeuwen, Phys. Rev. A.4, 2055(1971).

[16] J.R. Dorfman and E.G.D. Cohen, Phys. Rev. Lett. 25, 1257(1970).

[17] M.H. Ernst, Physica D 47, 198(1991).

[18] M.A. van der Hoef and D. Frenkel, Phys. Rev. A41, 4277(1990).

[19] M.A. van der Hoef and D. Frenkel, Physica D 47, 191(1991).

[20] M.A. van der Hoef and D. Frenkel, Phys. Rev. Lett. 66, 1591(1991).

[21] M.A. van der Hoef, M. Dijkstra and D. Frenkel, submitted for publication.

[22] P.-M. Binder and D. Frenkel, Phys. Rev. A41, 4277(1990).

[23] D. Frenkel, J. Phys.: Condens. Matter 2, SA265(1990).

[24] G.C.A.M. Mooij and D. Frenkel, Mol. Phys. (1991) in press.

[25] E.J. Meijer and D. Frenkel, Phys. Rev. Lett. (1991) in press.

[26] D.L. Ermak and J.A. McCammon, J. Chem. Phys. 69, 1352(1978).

[27] J.F. Brady and G. Bossis, Ann. Rev. Fluid Mech, 20, 111(1988).

[28] A.J. Masters, Mol. Phys. 57, 303(1986).

[29] J.A. Somers and P.C. Rem in : Proceedings of the Shell Conference on Parallel Computing, edited by G.A. van Zee (Lecture Notes on Computer Science 1988).

[30] A.J.C. Ladd and D. Frenkel in : Proceedings of workshop on Cellular Automata and Modelling of Complex Physical Systems, Les Houches, France, Feb. 21-28, 1989, edited by P. Manneville (Springer, Berlin, 1989).

[31] A.J.C. Ladd and D. Frenkel, Phys. Fluids A2, 1921(1990).

[32] A.J.C. Ladd in : Proceedings of NATO ASI on Computer Simulations in Material Science, Aussois, France, March 25 - April 5, 1991.

[33] T. Naitoh, M.H. Ernst and J.W. Dufty, Phys. Rev. A42, 7187(1990).

[34] M.A. van der Hoef, D. Frenkel and A.J.C. Ladd, submitted for publication.

[35] T.E. Wainwright, B.J. Alder and D. Gass, Phys. Rev. A4, 233(1971).

[36] A.F. Bakker, to be published. 